\title{
Correction to: Lung cancer and interstitial lung diseases: the lack of prognostic impact of lung cancer in IPF
}

\author{
Loredana Carobene ${ }^{1}$ (1) $\cdot$ Donatella Spina ${ }^{2} \cdot$ Maria Giulia Disanto ${ }^{2} \cdot$ Claudio Micheletto $^{1} \cdot$ Maria Antonietta Mazzei $^{3}$. \\ Piero Paladini ${ }^{4}$. Claudia Ghiribelli ${ }^{4}$ Elena Bargagli ${ }^{5} \cdot$ Paola Rottoli $^{5}$
}

Published online: 8 October 2021

(c) The Author(s) 2021

\section{Correction to: Internal and Emergency Medicine https://doi.org/10.1007/s11739-021-02833-6}

\section{Correction to: Fig. 1}

https://doi.org/10.1007/s11739-021-02833-6

The caption of Fig. 1 shown in the article is incomplete, the correct caption is shown below:

"Fig. 1 a Correlation between interval time 'LC diagnosis and follow up end' and DLCO\% in the three groups: UIP/IPF-LC (blue circles), SR-ILD-LC (green circles), O-ILD-LC (red circles). b Localization of lung cancer in the three groups: UIP/IPF-LC, SR-ILD-LC, O-ILD-LC. Blue columns: central cancer not in fibrotic area; green columns: peripheral cancer not in fibrotic area; beige columns: peripheral cancer in fibrotic area. c Survival in the three groups of patients: UIP/IPF-LC, SR-ILD-LC, O-ILD-LC. d Survival in patients with UIP/IPF with and without lung cancer (LC)".

Correction to: Abstract.

https://doi.org/10.1007/s11739-021-02833-6

The original article can be found online at https://doi.org/10.1007/ s11739-021-02833-6.

Loredana Carobene

loredana.carobene@aovr.veneto.it

$\triangle$ Claudio Micheletto

claudio.micheletto@univr.it

1 Cardio-Thoracic Department, Respiratory Unit, Verona Integrated University Hospital, University of Verona, Square Aristide Stefani, 1, Verona, Italy

2 Pathology Unit, Siena University Hospital, Siena, Italy

3 Diagnostic Imaging Unit, Siena University Hospital, Siena, Italy

4 Thoracic Surgery Unit, Siena University Hospital, Siena, Italy

$5 \quad$ Respiratory Disease and Lung Transplant Unit, Siena University Hospital, Siena, Italy
This phrase is incorrect: "Describe the association between lung cancer and ILD and evaluate the impact of LC on survival in these populations".

Below I reported the correct phrase:

"The objectives of this study are to describe the association between lung cancer and ILD and evaluate the impact of LC on survival in these populations."

I reported in abstract two abbreviations without explanation:

- "SR-ILD", I think is correct replaced it with "SR-ILD (smoking-related ILD)"

- "IPF", I think is correct replaced it with "IPF (idiopathic pulmonary fibrosis)".

\section{Correction to: Abbreviation:}

"IPF" is explained as "idiopathic interstitial pneumonia", the correct explanation is "IPF idiopathic pulmonary fibrosis";

The same error is present in other abbreviation, reported below:

- "UIP/IPF usual interstitial pneumonia/idiopathic interstitial pneumonia" that I think is correct to be replaced by "UIP/IPF usual interstitial pneumonia/ idiopathic pulmonary fibrosis".

- "UIP/IPF-LC usual interstitial pneumonia/idiopathic interstitial pneumonia with lung cancer" that I think is correct to be replaced by "UIP/IPF-LC usual interstitial pneumonia/idiopathic pulmonary fibrosis with lung cancer".

In another abbreviation there is an error:

- "TGF beta transforming growth factor beta" that I think is correct to be replaced by "TGF beta transforming growth factor beta". 
The original article has been corrected.

Publisher's Note Springer Nature remains neutral with regard to jurisdictional claims in published maps and institutional affiliations. 